\title{
The Application of Photorefractive Effect of Lithium Niobate in the Development of a Biometric Fingerprint Scanner with Unique Sensitivity
}

\author{
Uzoma I. Oduah
}

\begin{abstract}
This research includes the combination of the optical imaging and capacitive imaging to capture the biometric features of the fingerprint. The system therefore shuffles between the optical mode and capacitive mode while scanning. The photorefractive effect of Lithium Niobate is applied in holographic display of the fingerprint image. The developed fingerprint scanner offers improved diffraction efficiency, low power consumption, and maintains a high image resolution using the Volume Holographic Optical Correlator System.

The fingerprint scanner system comprises of the sensor for scanning fingerprint, a processor which stores the captured fingerprint database and a software which compares and matches the fingerprint using Fourier series. By capturing and matching the minutia features and fingerprint patterns, the scanner can authenticate a user.
\end{abstract}

Index Terms-Fingerprint, holography, photorefractive, imaging.

\section{INTRODUCTION}

The image capturing technique is very important in determining the effectiveness of biometric fingerprint scanner in authentication. The objective of the fingerprint scanner is to provide an identification of a person based on the acquisition and recognition of those unique patterns and ridges in fingerprint [1], [2]. The fingerprint is made up of a pattern of ridges and valleys (loop pattern, whorl pattern, and arch pattern) as well as characteristics that occur at the minutiae points classified as Ridge bifurcation, Short ridge or dot, and Ridge ending described in Fig. 1. The optical scanner captures the image of the fingerprint targeted on identifying the ridges and the valleys on the pattern of the finger placed on a glass plate using a Charged Couple Device (CCD) camera [3]-[5]. The capacitance scanner generates an image of the ridges and valleys of the finger using the active capacitive pixel sensing technology. Each sensor cell contains an active capacitive feedback circuit whose effective feedback capacitance is modulated by the presence of a live skin placed on the surface sensor.

Historically, there are various optical fingerprint recognition systems such as system based on Joint Transform Correlator and Volume Holographic Correlator, Fourier Plane Binary and Output Peak Intensity normalization technique. Other fingerprint recognition

Manuscript received January 15, 2014; revised March 18, 2014.

U. I. Oduah is with University Of Lagos, Physics Department, Lagos, Nigeria (e-mail: uoduah@unilag.edu.ng,uzoma_i_oduah@yahoo.com). system include Multi-channel matched correlators using photorefractive material that can output multiple correlation results with single input. Watanabe et al. proposed an ultrahigh speed compact optical correlation system using volume holographic disc that can be used for fingerprint recognition [6]-[10].

This research focuses on the capture of the fingerprint in 3-dimensional oriented ridge-valley pattern using a combination of capacitive sensor, optical sensor, and holography technology. Usually in fingerprint analysis, there are two kinds of singular points of key interest namely the cores and the deltas. In practice, the singular points detection and analysis is used for topographical feature of the fingerprint types. Most times the Point care index is used for singular points detection. This is defined as the sum of the orientation change along a close circle and around the points. However, due to the noise in the real images, this feature is not ideal for detection. A further development led to the discovery of multi-resolution approach. Other methods include the partitioning method and heuristic rules for detection. But all these works are only based on local characteristics of singular points. Consequently, the fingerprint analysis results are always affected by creases, scars, smudges, damped prints and other spurious detections [11]-[13]. An improvement of these methods is the application of orientation diffusion which uses the global constraint of the oriented texture during the dynamic diffusion process as proposed by Perona.

In this research, the combinational manifolds of dimensions $3(3 \mathrm{D}$ manifold) is used to deduce the relationship between the cores and deltas in the captured fingerprint. This process therefore implements an algorithm for both the local feature and the global information.

The application of Volume Holographic Imaging is very important in designing efficient application specific computational imaging systems where the hologram act as a front-end processor for the optical field, and the postprocessing algorithms, such as point multiplication and the pseudo-inverse, increase the information extracted from the raw image data.

The photorefractive effects of Lithium Niobate $\left(\mathrm{LiNb}_{3}\right)$ crystals which are characterized by a change in its refractive index that results from an optically induced separation of electrons and the linear electroptic effect have been used in various optical devices. The ability to record holograms makes $\mathrm{LiNbO}_{3}$ crystals attractive for many applications such as holographic data storage, optical information processing, phase conjugation, and wavelength filters. 


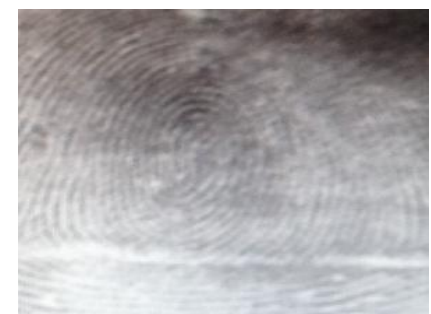

(a) The whorl

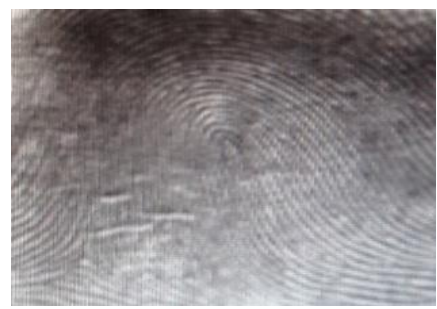

(b) The loop

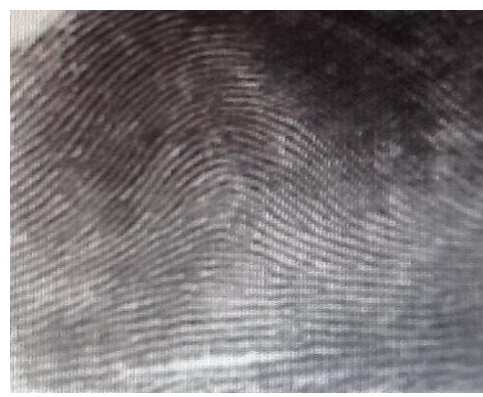

(c) The ARCH

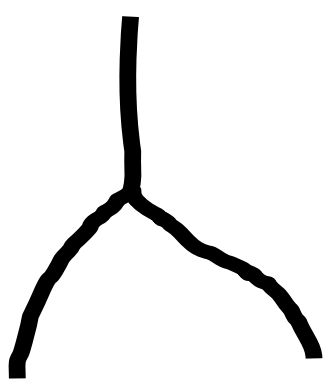

(d) Bifurcation

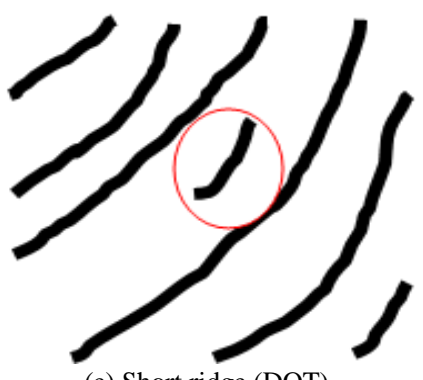

(e) Short ridge (DOT)

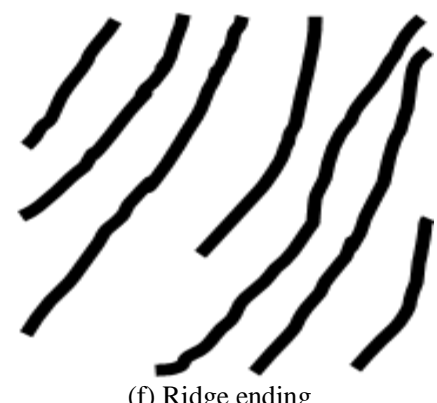

Fig. 1. The fingerprint patterns and minutia.

\section{THE FINGERPRINT SCANNER TECHNOLOGY}

The fingerprint scanner technology involves three processes namely the capturing of the fingerprint image, the storage of the captured image, and the sampling of the stored image for matching and retrieval. These processes must be properly implemented as any defect or imperfections will generally affect the efficiency and accuracy of the fingerprint scanner. A poorly captured image will introduce errors during sampling and retrieval of stored image. Also a bad storage device can lead to introduction of noise or even the loss of data. A faulty sampling and retrieval process will render inaccurate results with very poor sensitivity. It is therefore very important that all the steps are considered critically to achieve the desired efficiency.

\section{A. The Capturing of Fingerprint Image}

This research combines the capacitive method and the optical method to capture the fingerprint. A shutter controls the Laser beam which takes the image of the target first and shuts off before the capacitive sensor is enabled. The system shuffles between the capacitive mode and optical mode. During the optical mode, the main unit of the optical scanner is Charge Coupled Device (CCD). A CCD refers to an array of light sensitive diodes called photosites, which generate an electrical signal in response to light photons. So, the photosites record the pixels which are tiny dots representing the Laser beam that hit the finger being captured. A combination of the light and dark pixels constructs the image of the fingerprint as shown in Fig. 2. A system of analogue to digital converter processes the analogue electrical signal to generate a digital representation which is then matched with the signal transmitted from the capacitive method.

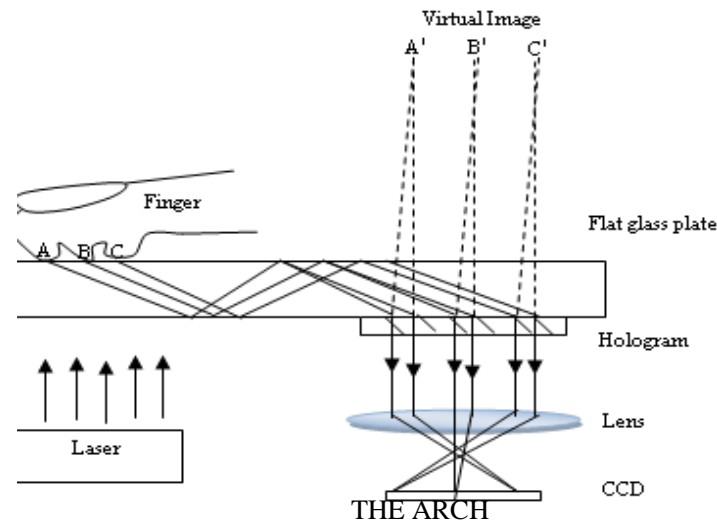

Fig. 2. Optical scanner.

In the capacitive mode, the surface of each pixel is composed of two adjacent metal plates which are separated from the skin and the environment by an ultra hard protective coating. These sensor plates create a fringing capacitance between them whose field lines extend beyond the surface of the silicon as shown in Fig. 3. When the finger is placed close to the sensor plates, the fingerprint valley and ridge interferes with the field lines between the two plates and reduces the effective capacitance between them. The feedback capacitance is minimized at the fingerprint ridge while the feedback capacitance is maximized at the fingerprint valley. The action of the sensor 
cell is in two phases namely the reset phase and the set phase. During the reset phase, the input and the output of the inverter are shorted together through a reset switch, causing the charge integrator output to settle to the logical threshold of the inverter. In the set phase, the reset switch is opened and a calibrated charge is input into the input plate of the sensor cell, causing the charge integrator output to change by an amount proportional to the feedback capacitance between the two sensor cells. As the feedback capacitance of a fingerprint ridge is smaller than that of a fingerprint valley, consequently the output swing of a sensor cell under a ridge will be greater than the output swing of a sensor cell underneath a fingerprint valley. A two dimensional array of sensor cells is used to capture the entire fingerprint image which is addressed in a random access mode through row and column decoders. Now, using advanced functions of windowing and sub-sampling, the output of the sensor array is passed through an analoguesignal conditioning block providing the capability to adjust sensor gain and offset before the signal is converted through on-chip Analogue-Digital Converter into an 8-bit digital signal for output off chip.

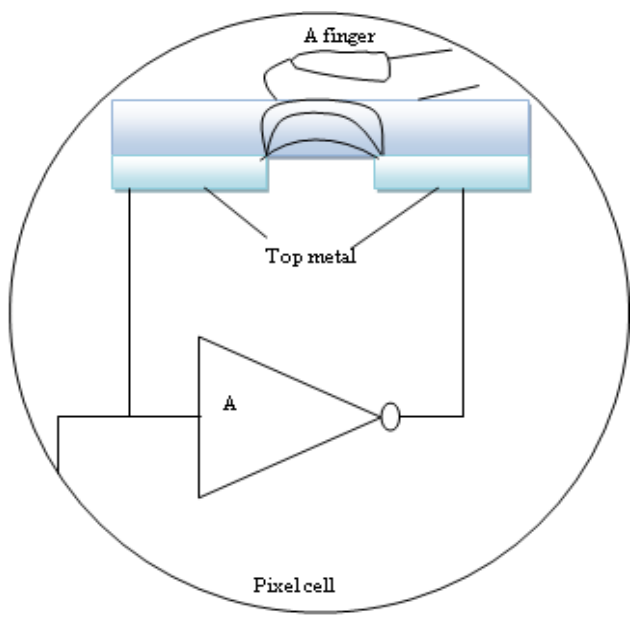

Fig. 3. The capacitive sensor.

The template data page construction was done with Minutiae Cylinder-Code (MCC) described in Fig. 5. This fingerprint encoding method is named as multi-page local structure coding with all the parameters described in Fig. 5. A fingerprint image is assigned $N_{m}$ minutiae a template data page with $\mathrm{Nm}$ equals to the total number of minutiae of that fingerprint [14], [15]. The accumulating contribution of neighboring minutiae around the cell

$\left(m_{t} \in N_{p i j}\right)$ is defined as:

$$
V(i, j, k)=\sum m_{t} \in N_{p i j}\left(C_{M}^{S}\left(m_{t}, p_{i, j}\right) \cdot C_{M}^{D}\left(m_{t}, \emptyset_{k}\right)\right)
$$

where $C_{M}^{S}\left(\mathrm{~m}_{\mathrm{t}}, \mathrm{p}_{\mathrm{i}, \mathrm{j}}\right)$ and $C_{M}^{D}\left(\mathrm{~m}_{\mathrm{t}}, \emptyset_{\mathrm{k}}\right)$ are the distance and angle contribution minutia $m_{t}$ gives to cell $(i, j, k)$

\section{B. Storage of Fingerprint Templates}

There are four major locations for storing of a template as follows: In a token or smartcard; in a central database on a server; on a workstation; or directly on the sensing device. Each of the locations offers its unique applications with advantages and disadvantages. Fingerprint stored on a portable token such as smartcards can be used for identity card purposes. It enables the user to carry the information from one point to another. It requires the encryption of the fingerprint code on a chip which can be decoded by a reader device. Large storage templates can be done on a central repository on a server. This allows multiple-access and with sufficient memory size can contain fingerprint templates for numbers above trillions. It is very suitable for national census data collection and other public data centre. Individual workstation is a middle ground between central database and storage on a sensing device. With workstation the user cannot authenticate from multiple locations.

\section{The Fingerprint Image Sampling and Retrieval Technique}

The Volume Holographic Optical Correlator System was used for the recognition system as described in Fig. 4 .

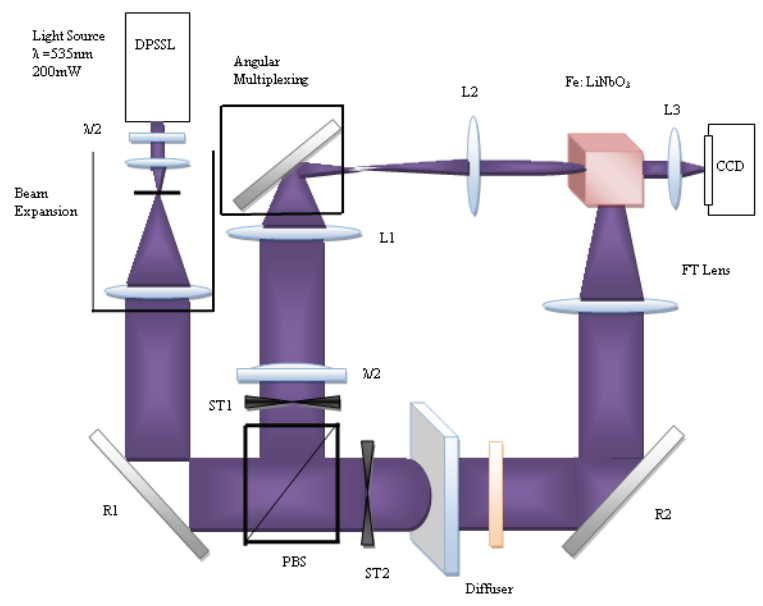

Fig. 4. Volume holographic optical correlator system.

First step: The minutiae of each fingerprint extracted by the optical and capacitive method are constructed into Minutiae Cylinder Code. Secondly, the encoded data pages of the database are sequentially stored into $\mathrm{Fe}: \mathrm{LiNbO}_{3}$ crystal applying angular multiplexing.

Third Step: Search arguments are created by uploading all the query fingerprints to the Spatial Light Modulator (SLM). Finally, the fingerprint matching scores are calculated based on similarities in the minutiae samples [16]-[21].

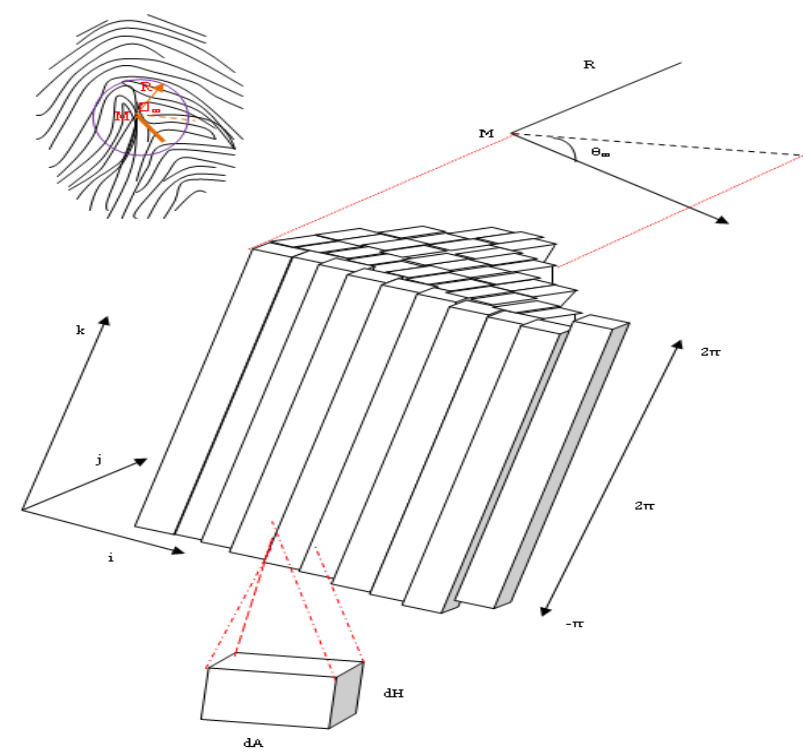

Fig. 5. Describling the system for minutiae cylinder code (MCC). 


\section{RESULTS}

A Biometric fingerprint scanner that shuffles between the optical mode and capacitive mode has been developed. The photorefractive effect of Lithium Niobate was applied in the holographic display of the fingerprint image. The developed fingerprint scanner was subjected to robustness test and sensitivity test. The developed fingerprint scanner was found to detect gummy fingers and artificial fingers made with silicon or gelatine.

\section{CONCLUSION}

The development of a fingerprint scanner requires an image capturing technique that is devoid of trapezoid distortions and all forms of imperfections experienced in conventional fingerprint sensors. The application of volume holographic imaging using the photorefractive effect of Lithium Niobate improved the efficiency of the fingerprint device by $98 \%$.

\section{ACKNOWLEDGEMENT}

My gratitude goes to Prof. E. I. E. Ofodile, Dean of Faculty of Engineering and Technology, Nnamdi Azikiwe University Awka, Nigeria, and the Photonics Research Group of Physics Department, University of Lagos, Nigeria.

\section{REFERENCES}

[1] M. Kawagoe and A. Tojo, "Fingerprint pattern classification," Pattern Recognition, vol. 17, pp. 295-303, 1984.

[2] A. Shimisu and M. Hase, "Entry method of fingerprint image using a prism," Trans. Inst. Electron. Commum. Eng. Jpn. vol. 67-D, pp. $627-$ 628, 1984.

[3] D. Maltoni, D. Maio, A. K. Jain, and S. Prabhakar, Handbook of Fingerprint Recognition, Springer, 2009, ch. 4, pp. 167-233.

[4] J. Dai, J. Feng, and J. Zhou, "Robust and efficient ridge-based palmprint matching," IEEE Trans. Pattern Anal Mach. Intell., vol. 34, no. 8, pp. 1618-1632, 2012.

[5] X. Jiang and W. Y. Yau, "Fingerprint minutiae matching based on the local and global structures," in Proc. the $15^{\text {th }}$ International Conference on Pattern Recognition 2, Institute of Electrical and Electronics Engineers, New York, 2000, pp. 1038-1046.

[6] P. A. Mitkas and G. W. Burr, "Volume holographic optical correlators," in Holographic Data Storage, H. J. Coufal, D. Psaltis, and G. T. Sicebox, Eds. Springer-Verlag, 2000, pp. 429-451.

[7] B. V. Kumar, A. Mahalanobis, and R. D. Juday, Correlation Pattern Recognition, Cambridge University, 2005, ch. 8, pp. 295-360.

[8] K. H. Fielding, J. L. Horner, and C. K. Makekau, "Optical fingerprint identification by binary joint transform correlation," Opt. Eng., vol. 38, no. 12, pp. 1958-1962, 1991.

[9] E. Watanabe, A. Naito, and K. Kodate, "Ultrahigh-speed compact optical correlation system using holographic disc," in Proc. SPIE 7442, 2009, pp. 1493-1495.

[10] S. H. Lee, S. Y. Yi, and E. S Kim, "Fingerprint identification by use of a volume holographic optical correlator," in Proc. SPIE 3715 , 1999 , pp. 321-330.
[11] N. K. Ratha, R. M. Bolle, V. D. Pandit, and V. Vaish, "Robust fingerprint authentication using local structural similarity," in Proc. the $5^{\text {th }}$ IEEE Workshop on Applications of Computer Vision, 2000, pp. 29-31.

[12] J. Feng, "Combining minutiae descriptors for fingerprint matching," Pattern Recognition, vol. 41, no. 1, pp. 342-355, 2008.

[13] A. A. Paulino, J. Feng, and A. K. Jain, "Latent fingerprint matching using descriptor-based hough transform," IEEE Trans. Inf. Foren. Sec., vol. 8, no. 1, pp. 31-48, 2013.

[14] T. J. Grycewicz, "Techniques to improve binary joint transform correlator performance for fingerprint recognition," Opt. Eng., vol. 38, no. 1, pp. 114-120, 2005.

[15] Y. Yan, G. Huang, W. Feng, G. Jin, and M. Wu, "Multichannel wavelet correlators for fingerprint identification by the use of associative storage in a photorefractive material," in Proc. SPIE 3458, 1998, pp. 259-270.

[16] C. M. Vest, "Formation of images from projections: Randon and abel transforms," J. Opt. Soc. Am., vol. 64, no. 9, pp. 1215-1220, 1974.

[17] P. J. V. Heerden, "Theory of optical information storage in solids," Appl. Opt., vol. 2, no. 4, pp. 393-411, 1963.

[18] J. W. Goodman, Introduction to Fourier Optics, 2nd ed., New York: McGraw Hill, 1996.

[19] D. L. Marks, R. A. Stack, D. J. Brady, D. C. Munson Jr., and R. B Brady, "Visible cone-beam tomography with a lensless interferometric camera," Science, vol. 284, no. 5423, pp. 2164-2168, 1999.

[20] P. Yeh, Introduction to Photorefractive Nonlinear Optics, New York: Wiley \& Sons, 1993

[21] M. P. Bernal, G. W. Burr, H. Coufal, R. K. Grygier, J. A. Hoffngale, C. M. Jefferson, R. M. Shelby, G. T. Sincerbox, and G. Wittmann, "Holographic-data-storage materials," MRS Bull., vol. 21, no. 9, pp. 51-62, 1996

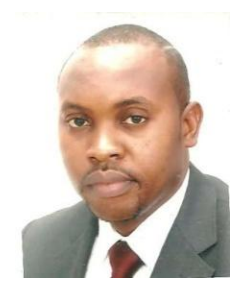

Uzoma I. Oduah was born in Ogwashi-Uku, Nigeria, on October 31, 1971.

He holds both a doctorate degree (Ph.D.) in physics electronics in 2009 and a master of technology degree (M. Tech.) in physics electronics in 2000 from Nnamdi Azikiwe University, Awka, Anambra State, Nigeria. Furthermore, he specializes in nanotechnology, semiconductor bandgap engineering, photonics and advanced micro-

electronics.

He is a lecturer in Physics Department of University of Lagos, Faculty of Sciences, Akoka Campus, Lagos, Nigeria where he is involved in teaching some of the departmental courses in optoelectronics, advanced digital electronics, photonics, and laboratory practical demonstrations. He has over 10 years research experience garnered from various world class institutions. His previous work experience includes a business development manager, in telecommunication group of Spring Bank Plc, 143 Ahmadu Bello way, Victoria Island, Lagos, Nigeria in 2010 where he rendered professional advisory services to telecommunication multinational companies. His current research work is on carrier mobility enhancement in semiconductor complementary heterostructure metal oxide semiconductor field effect transistors (CHMOSFET)

Dr. Oduah is a fellow of Materials and Photonics Research Centre (MAPHORC) University of Lagos, Nigeria. He is a senior member of International Association of Computer Science and Information Technology (IACSIT). Also, he is a member of Biometric Research Professionals and a Member of Institute of Physics (IOPs) International. 